\title{
Kimura's Disease - A Rare Cause of Neck Swelling
}

\author{
N Dinesh K ${ }^{1}$, A Manoharan², Kumaran $\mathbf{A A}^{3}$, S Balaji $^{4}, \mathbf{S}$ Sivaraman $^{5}$ \\ ${ }^{1}$ Dr N Dinesh Kumar, Assistant Professor, Department of Pediatrics, ${ }^{2}$ Dr A Manoharan, Post Graduate student in Pathology, \\ ${ }^{3}$ Dr. A Arul Kumaran, Associate Professor, Department of Pediatrics, ${ }^{4}$ Dr. S Balaji, Post Graduate in Pediatrics, ${ }^{5}$ Dr. S \\ Sivaraman, Post Graduate in Pediatrics. All are affiliated to Sri Manakula Vinayagar Medical College and Hospital, \\ Puducherry, India.
}

Address for correspondence: Dr N Dinesh Kumar, Email: dinesh.paed@gmail.com

\begin{abstract}
Kimura's disease is a rare form of chronic inflammatory disorder typically affecting Asian males of 30 to 40 years of age (male : female ratio is 3:1). Here we report the case of a 12 year old Asian girl with chronic neck swelling affected by this uncommon disease with extremely rare presentation in this age and sex.
\end{abstract}

Key words: Eosinophilia, Kimura’s Disease, Lymphadenopathy, Serum Immunoglobin E.

\section{Introduction}

Kimura's disease is a rare chronic inflammatory disorder of unknown aetiology, characterised by inflammation of salivary glands, lymph nodes and sub cutaneous tissue [1] and usually associated with blood and tissue eosinophilia with increased serum Immunoglobin E level. Worldwide only 120 cases of kimura's disease have been reported [2]. Even though they are benign, their unknown cause and unpredictable response to therapeutic intervention make them a challenge to the treating physician [3].

\section{Case Report}

A well-nourished 12 year old girl reported to our Out Patient Department with a history of slowly enlarging painless swelling on left side of neck for past 8 months, not associated with fever, weight loss, cough, loss of appetite and night sweats. On examination the girl weighed appropriate for age, not pale and had no generalised lymphadenopathy. Local examination showed a $3 * 5 \mathrm{~cm}$ firm non tender swelling behind the angle of the mandible, not lifting the left ear lobe. The swelling was smooth; skin over the swelling was normal and pinchable. No other lymph node enlargement in the neck. Her oral cavity, dentition and oropharynx were normal. Examination of other systems were unremarkable and no hepatosplenomegaly. Based on these grounds child was suspected and evaluated for lymphoma and tuberculosis

Manuscript received: $11^{\text {th }}$ Oct 2014

Reviewed: $16^{\text {th }}$ Oct 2014

Author Corrected; $29^{\text {th }}$ Oct 2014

Accepted for Publication: $10^{\text {th }}$ Nov 2014
The initial investigations revealed Haemoglobin - 11 $\mathrm{gm} / \mathrm{dL}$, total count of $11,000 / \mathrm{cu} \mathrm{mm}$, differential count of Polymorphs - 32\%, lymphocytes - $28 \%$, eosinophil -40 $\%$ ( Absolute eosinophil count - 4400 ) and ESR $30 \mathrm{~mm} /$ hour. Urine albumin - Nil, no mediastinal widening in Chest $\mathrm{X}$ ray, Mantoux skin testing negative, ultra sonogram of abdomen showed no hepatsplenomegaly, no mesenteric lymphadenopathy. Serum Immunoglobulin E level was raised significantly to $512 \mathrm{IU} / \mathrm{ml}$. In this clinical setting we suspected Kimura's disease and went for excision biopsy as FNAC was inconclusive.

Histopathological examination of the biopsy specimen revealed prominent lymphoid hyperplasia with germinal center proliferation, part of the lymphoid follicles showing prominent eosinophilic infiltration with few mast cells and capillaries ( Fig 1 ), eosinophilic microabscess ( Fig 2 ) foci of follicles showing folliculolysis, hyalinized vessels and proteinaceous material ( Fig 3 ) and part of the lymph node showing sclerosis around the lymphoid follicles ( Fig 4 ).

After discussing about various treatment modalities, the parents chose oral corticosteroids therapy for their girl. The girl was discharged on oral prednisolone $(1 \mathrm{mg} / \mathrm{kg} /$ day) for 1 month, during review the girl showed decrease in absolute eosinophil count and regression of size of the swelling. Steroid was tapered and stopped after 1 month, in 3 month follow up there was no recurrence. After 3 months she developed recurrence at same site and their parents were not willing for either alternative drugs or intra lesional steroid or definitive surgery. They were 


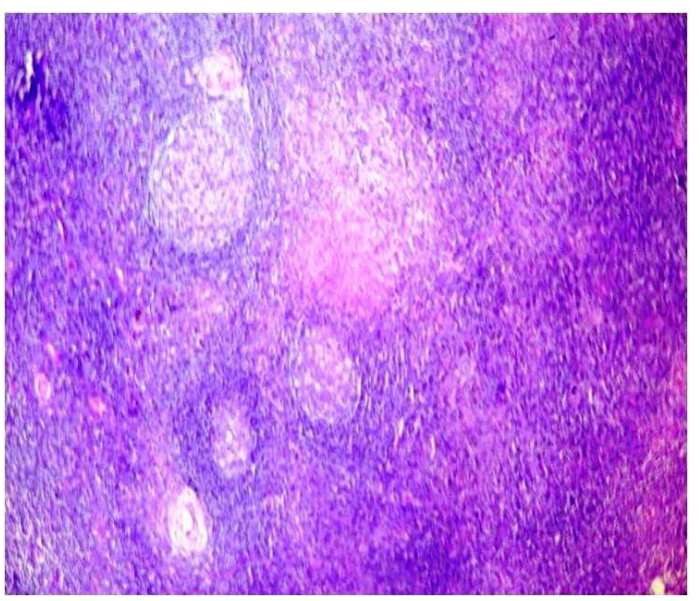

Fig 2: Lymphoid follicle with eosinophilic microabscess (H \& E 4x)

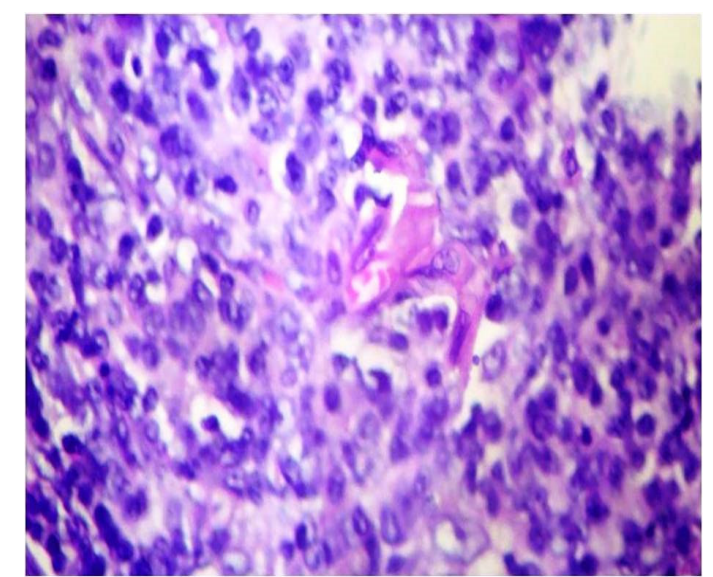

Fig 4: Sclerosis around lymphoid follicle (H\&E 40x)

\section{Discussion}

Kimura's disease was first reported from China in 1937 by Kimm and Szeto. as "eosinophilic hyperplastic granuloma" . It was named after Kimura who reported it as "eosinophilic hyperplastic granuloma" in 1948 [1]. It mainly affects the young Asian men (male: female is $3: 1$ ).

Kimura's disease is an idiopathic chronic inflammatory condition characterised by a triad of painless subcutaneous swelling in head and neck region, eosinophilia and elevated serum Immunoglobin E levels [5]. The disease is classified as a benign reactive process possibly due to allergic, infections, auto immune stimuli.

Peripheral blood eosinophilia and an elevated serum immunoglobulin E (Ig E) levels are consistent laboratory findings in Kimura's disease. Associated renal disease is found in $12 \%$ of Kimura's and they are characterised by proteinuria [6]. 


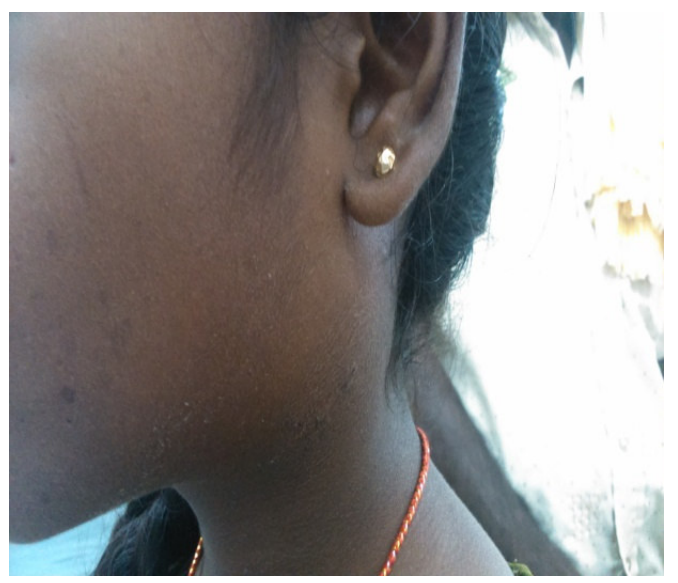

Fig 5: painless swelling on left side of neck

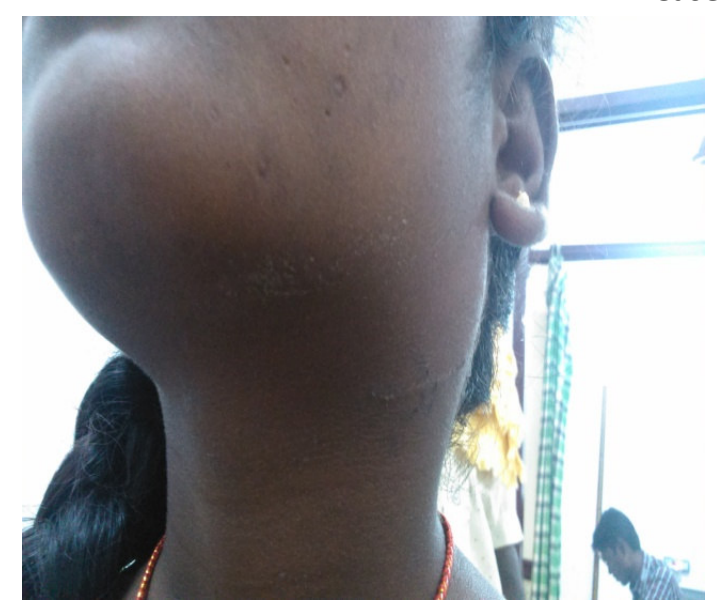

Fig 5: painless swelling on left side of neck
The common histological findings seen in Kimura are preserved lymphnode architecture, florid germinal centre, eosinophilic infilterates and increased number of post capillary venules. The frequent findings include sclerosis, karyocytolysis in both germinal centre and para -cortical region, vascularisation of the germinal centres in the sclerotic area [7].

The important differential diagnosis for Kimura's disease is Angiolymphoid Hyperplasia with Eosinophilia (ALHE). The common feature shared between them are male preponderance, common in head and neck region, tendency to recur and vascular nature of the lesion with lymphoid and eosinophilic infiltrates. The differentiating feature of ALHE lesions are multiple dermal papular or nodular eruption in older patients not frequently associated with eosinophilia. The main histologically differentiating feature is presence of histiocytoid and epithelioid blood vessels in ALHE, which is lacking in Kimura's disease [8].

The treatment of choice is said to be surgical excision. Although alternative treatment including radiation, high dose intra lesional steroids and vinblastine have been attempted with good response, they recur on stopping the treatment. Some have reported satisfactory results with cyclosporine and pentoxifylline $[9,10]$.

\section{Conclusion}

This report highlights the importance of having Kimura's disease as one of the differentials while evaluating lymphadenopathy particularly when associated with increased eosinophils. High index of suspicion is needed for early diagnosis and appropriate treatment.

Funding: Nil

Conflict of interest: Nil

Permission from IRB: Yes

\section{Reference}

1. Kimura $\mathrm{T}$, Yoshimura $\mathrm{S}$, Ishikaura E. Unusual granulation combined with hyperplastic changes of lymphatic tissue. Trans Soc Patho Jpn 1948;37:179-80.

2. Armstrong WB, Allison G, Pena F, Kim JK. Kimura's disease: two cases and literature review. Ann Otol Rhinol Laryngol. 1998 Dec;107(12):1066-71.

3. Jani A, Coulson M, Kimura's disease: Atypical case of a rare disorder. West J Med 1997;166:142-44.

4. Irish JC, Kain K, Keystone JS et al Kimura's disease an unusual cause of Head and Neck masses. Journal of Otolarngology 1994 ; 23:88-91.

5. Cecen E, Kacar-Doger F, Etensel B. An extremely rare cause of generalised lymphadenopathy in children: Kimura's disease. Turk J Pediatr 2010;52:534-7.

6. Yamada A, Mitsuhashi K, Miyakawa Y, et al (1982) Membranous glomerulonephritis associated with eosinophillic lymphofolliculosis of the skin (Kimura's disease): Report of a case and review of the literature. Clin Nephrol 18:211-215.

7. Kumar V, Salini, Haridas S. Kimura's disease : An uncommon cause of lymphadenopathy. Indian $J$ Med Paediatr Oncol 201;31:89-90.

8. Kuo TT, Shih LY, Chan HL (1998) Kimura's disease : Involvement of regional lymph nodes and distinction form angiolymphoid hyperplasia with eosinophilia. Am J Surg Pathol 12:843-854. 
9. Wang YS, Tay YK, Tan E, Poh WT. Treatment of Kimura's disease with cyclosporine. J Dermatolog Treat 2005;16:242-44.
10. Hongcharu W, Baldassano M, Taylor CR. Kimura's disease with oral ulcers : response to pentoxifylline. $J \mathrm{Am}$ Acad Dermatol 2000;43:905-907.

\section{How to cite this article?}

N Dinesh K, A Manoharan, Kumaran AA, S Balaji, S Sivaraman .Kimura's Disease - A Rare Cause of Neck Swelling . Pediatr Rev: Int J Pediatr Res 2014;1(2):61-64. doi:10.17511/ijpr.2014.102.05 